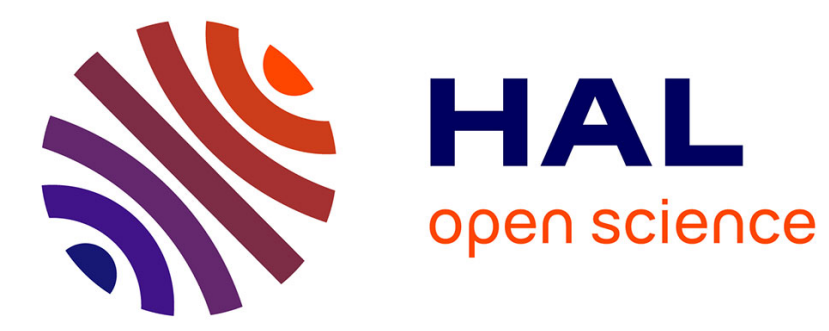

\title{
Investigating the effect of DMRI signal representation on fully-connected neural networks brain tissue microstructure estimation
}

\author{
Mauro Zucchelli, Samuel Deslauriers-Gauthier, Rachid Deriche
}

\section{- To cite this version:}

Mauro Zucchelli, Samuel Deslauriers-Gauthier, Rachid Deriche. Investigating the effect of DMRI signal representation on fully-connected neural networks brain tissue microstructure estimation. ISBI 2021 - 18th IEEE International Symposium on Biomedical Imaging, Apr 2021, Nice / Virtual, France. 10.1109/ISBI48211.2021.9434046 . hal-03174220

HAL Id: hal-03174220

https://hal.inria.fr/hal-03174220

Submitted on 19 Mar 2021

HAL is a multi-disciplinary open access archive for the deposit and dissemination of scientific research documents, whether they are published or not. The documents may come from teaching and research institutions in France or abroad, or from public or private research centers.
L'archive ouverte pluridisciplinaire HAL, est destinée au dépôt et à la diffusion de documents scientifiques de niveau recherche, publiés ou non, émanant des établissements d'enseignement et de recherche français ou étrangers, des laboratoires publics ou privés. 


\title{
INVESTIGATING THE EFFECT OF DMRI SIGNAL REPRESENTATION ON FULLY-CONNECTED NEURAL NETWORKS BRAIN TISSUE MICROSTRUCTURE ESTIMATION
}

\author{
Mauro Zucchelli, Samuel Deslauriers-Gauthier, Rachid Deriche \\ Université Côte D’Azur, Inria, France
}

\begin{abstract}
In this work, we evaluate the performance of three different diffusion MRI (dMRI) signal representations in the estimation of brain microstructural indices in combination with fully connected neural networks (FC-NN). The considered signal representations are the raw samples on the sphere, the spherical harmonics coefficients, and a novel set of recently presented rotation invariant features (RIF). To train FC-NN and validate our results, we create a synthetic dMRI dataset that mimics the signal properties of brain tissues and provides us a real ground truth for our experiments. We test 8 different network configurations changing both the depth of the networks and the number of perceptrons. Results show that our new RIF are able to estimate the brain microstructural indices more precisely than the diffusion signal samples or its spherical harmonics coefficients in all the tested network configurations. Finally, we apply the best-performing FC-NN in-vivo on a healthy human brain.
\end{abstract}

Index Terms - Spherical Harmonics, Rotation Invariant Features, Neural Networks

\section{INTRODUCTION}

One of the main diffusion Magnetic Resonance Imaging $(\mathrm{dMRI})$ research topic is the estimation of brain tissue microstructure in-vivo. This is normally done by fitting the dMRI signal with bio-physical models that mimic the brain microstructure with understandable parameters that characterize the different tissues. These models simulate the water diffusion in the different cellular compartments (intraaxonal, extra-axonal, etc.) and are therefore called multicompartment models [1, 2, 3]. However, finding the model parameters require a non-linear fitting of the dMRI signal with the models which is non-trivial. Several techniques have been proposed to improve the accuracy of the fitting [2] 3]. The dMRI signal can be viewed as a spherical function and most of these techniques rely on converting the diffusion signal into a rotation invariant representation. Recently, we proposed a new framework for estimating a complete set of algebraically independent rotation invariant features (RIF) from the dMRI signal Spherical Harmonics ( $\mathrm{SH}$ ) series ex- pansion [4]. Their use was shown to improve the result of the multi-compartment models fitting.

Neural networks have been introduced to the dMRI community in recent years, mainly for disease classification tasks [5]. A first example of the use of fully-connected neural networks (FC-NN) to the estimation of microstructural indices is represented by the work of Golkov and colleagues in 2016 [6]. In Golkov et. al. (2016) the network was blind to the spherical nature of the dMRI signal, and raw dMRI samples were vectorized and used as the input of FC-NN to estimate three microstructural indices. The network was trained using the result of the in-vivo fitting of the neurite orientation dispersion and density imaging model [1].

In this work, we propose to investigate how three dMRI signal representations perform in the estimation of microstructural indices when used as an input for FC-NN. The three signal representation are the vectorized signal samples as in [6], the signal SH coefficients, and our new RIF. In the next Section we will briefly introduce the new RIF, the synthetic dataset used for the training and the validation of the FC-NN, and the architecture of the FC-NN themselves.

\section{MATERIAL AND METHODS}

\subsection{Rotation invariant features}

In this work, we consider the dMRI signal at a given b-value as a real-valued antipodal symmetric spherical function $f(\mathbf{u})$ parameterized by the $3 \mathrm{D}$ unit vector $\mathbf{u}$. Its $\mathrm{SH}$ series expansion can be expressed as

$$
f(\mathbf{u})=\sum_{l=0, \text { even }}^{L} \sum_{m=-l}^{l} c_{l m} Y_{l}^{m}(\mathbf{u})
$$

where $Y_{l}^{m}$ are the real SH [7] of degree $l$ and order $m, c_{l m}$ are the $\mathrm{SH}$ coefficients, and $L$ is the truncation degree of the $\mathrm{SH}$ series. In our previous work [4], we defined a new set of RIF based on the SH expansion of the dMRI signal whose take the form

$$
I_{\mathbf{l}}[f]=\sum_{m_{1}=-l_{1}}^{l_{1}} \ldots \sum_{m_{d}=-l_{d}}^{l_{d}} c_{l_{1} m_{1}} \cdots c_{l_{d} m_{d}} G\left(l_{1}, m_{1}|\cdots| l_{d}, m_{d}\right)
$$


where $G$ represents the generalized Gaunt coefficients, as the integral of $d \mathrm{SH}[7]$ and $\mathbf{l}=\left[l_{1}, l_{2} \cdots, l_{d}\right]$. For any set of $\mathbf{l} \mathrm{Eq}$. (2) will be rotation invariant for the signal described by the SH coefficients $c_{l m}$. In [4] we used the algorithm proposed by Caruyer and Verma (2015) [8] to select the $I_{1}$ that form a set of algebraically independent invariants to maximize the amount of information extracted from the signal while reducing the number of invariants. In this work, we consider the SH expansion of the dMRI signal of degree $L=4$ which corresponds to 12 algebraically independent invariants [4].

\subsection{Synthetic and in-vivo dataset}

We generate the dMRI signal using the Human Connectome Project (HCP) diffusion MRI sampling scheme [9]. It is composed of 18 samples $b$-value $0 \mathrm{~s} / \mathrm{mm}^{2}$, and three shells with 90 samples per shell at $b$-values 1000,2000 , and $3000 \mathrm{~s} / \mathrm{mm}^{2}$, respectively. We simulate our data using a multi-compartment model [1, 2, 3] for modeling a synthetic white matter fiber composed of an intra-axonal part modeled as a stick and an extra-axonal part modeled as an axially symmetric Gaussian. More in detail, we model the diffusion signal $E$ funtion of the $b$-value and the diffusion gradient direction $\mathbf{u}$ as

$E(b, \mathbf{u})=\nu_{i a} e^{-b\left(\lambda_{\|}\left(\mathbf{u}^{T} \mathbf{v}\right)^{2}\right)}+\left(1-\nu_{i a}\right) e^{-b\left(\left(\lambda_{\|}-\lambda_{\perp}\right)\left(\mathbf{u}^{T} \mathbf{v}\right)^{2}+\lambda_{\perp}\right)}$

where the parameters of the model are the signal fraction of the intra-axonal compartment $\nu_{i a}$, the parallel diffusivity $\lambda_{\|}$ which we considered the same for both the intra and extra compartments, and the extra-axonal perpendicular diffusivity $\lambda_{\perp}$. Eq. (3) represents the signal of a single synthetic fiber aligned in the direction $\mathbf{v}$. In order to simulate crossings, each synthetic voxel is composed of three randomly oriented fibers, all three having the same microstructural parameters. We also blurred each of the fiber using the Watson distribution [1] with random dispersion to mimic the natural undulation and dispersion of the brain fibers. For the microstructural parameters, we select $\nu_{i a}$ in the range $[0,1], \lambda_{\|}$in the range $[0.5,3] \times 10^{-3} \mathrm{~mm}^{2} / \mathrm{s}$, and $\lambda_{\perp}$ in the range $\left[0, \lambda_{\|}\right]$to preserve the zeppelin shape of the extra-axonal compartment. We simulate 300000 synthetic voxels adding noise with a signal-tonoise ratio of 30 . In order to test our methods in-vivo, we additionally consider a subject of the HCP dataset. Each HCP subject is composed of a volume $145 \times 174 \times 145$ voxels with a $1.25 \times 1.25 \times 1.25 \mathrm{~mm}^{3}$ resolution. We normalized the diffusion signal in the $[0,1]$ range dividing the diffusion signal by the mean value of the $b$-value $0 \mathrm{~s} / \mathrm{mm}^{2}$ samples.

\subsection{Neural Networks design}

In order to evaluate the microstructural parameters estimation potential of our RIF, we created 8 different neural networks. Each of these networks takes as input the 12 RIF estimated on each shell of the dMRI signal, for a total of 36 input channels considering the three b-values of the HCP scheme. The number of hidden layers $n_{h}$ and the number of perceptrons per layer $n_{p}$ was varied for each network. More specifically, we select $n_{h}$ equal to $[2,4]$ and $n_{p}$ equal to $[16,32,64,128]$ for a total of 8 networks. We refer to these FC-NN that take as input the 12 invariants, as RIF-NET. We also considered similar networks for the dMRI signal and the SH coefficients. The dMRI signal networks (SIG-NET) take as input the normalized diffusion signal in each shell, in our case 90 samples times three b-values, for a total of 270 input channels. The $\mathrm{SH}$ coefficients networks (COEF-NET) take the 15 coefficients of $\mathrm{SH}$ of degree 4 as input for a total of 45 channels. All the aforementioned networks have three channels as output for the three considered microstructural parameters, namely the intra-axonal signal fraction, the parallel diffusivity, and the perpendicular diffusivity. Since the number of input channels differs, the three sets of networks have a different number of weights even if $n_{h}$ and $n_{p}$ are the same, with SIG-NET having more tunable network weights than COEF-NET and RIFNET. However, for the sake of simplicity, we will consider these networks equivalent in potential and comparable given the fact that they have the same number of hidden layers and perceptrons.

The code of this work, including also the synthetic data generation, has been written in Python and it is available on request. All the networks are implemented in Pytorch ${ }^{1}$ and trained using the Adam optimizer with Mean Square Error loss with a learning rate of $5 \times 10^{-5}$ and a weight decay of $1 \times 10^{-6}$. We split the training set in batches of 100 elements and train the networks for 100 epochs. All these parameters were fine-tuned based on the global results of the three sets of networks and only the resulting best parameters are shown in this work. Of the 300000 synthetic voxels, 100000 were used for training the networks which will be used to calculate the microstructural feature for the full synthetic dataset and the in-vivo HCP subject.

\section{RESULTS}

To test the performance of our networks we calculate the mean absolute error between the ground truth microstructural parameters used for generating the signal and the value given in output by each network. In order to keep all the measures in the same scale, we divided $\lambda_{\|}$and $\lambda_{\perp}$ by $3 \times 10^{-3} \mathrm{~mm}^{2} / \mathrm{s}$ to keep their values between zero and one. Figure 1 illustrates the error of the estimation of the three microstructural parameters as a function of the number of perceptrons $n_{p}$ for the networks with $n_{h}=2$ (top row) and with $n_{h}=4$ (bottom row). For all the tested network configurations, RIF-NET (blue triangles) outperformed both SIG-NET (red circles) and COEF-NET (blue crosses). SIG-NET shows a lower error than COEF-NET for small networks $\left(n_{h}=2\right)$ but a higher error for bigger networks $\left(n_{h}=4\right)$. As expected, each

1 https://pytorch.org/ 

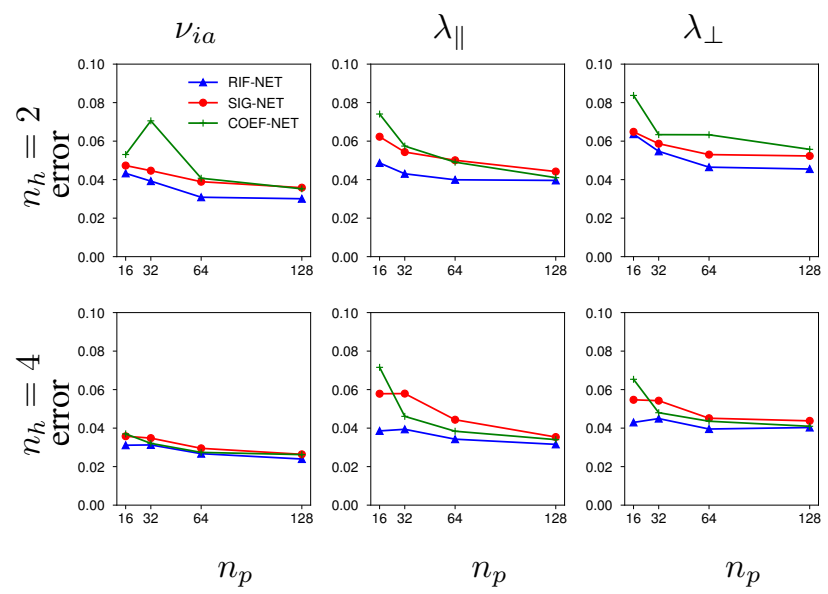

Fig. 1. Mean absolute error for the three microstructural features obtained for the two hidden layer networks $\left(n_{h}=2\right.$, top row) and the four hidden layer networks $\left(n_{h}=4\right.$, bottom row) for RIF-NET, SIG-NET, and COEF-NET.

increase in the number of learnable network weights leads to a decrease in the errors. This phenomenon is consistent for the three families of FC-NN, both when we increase the number of hidden layers and when we increase the number of perceptrons per layer. All the neural networks seem to reach a plateau in the three parameters errors while increasing the number of network weights. In fact, the difference between the performance of RIF-NET, SIG-NET, and COEF-NET tends to reduce with the increase of the network size. Overall the best performing network is the RIF-NET with $n_{h}=4$ and $n_{p}=128$.

Figure 2 illustrates the result of the estimation of the microstructural parameters for the best performing networks for one HCP subject. Although the network processes each voxel independently the microstructural parameters images look smooth while keeping the characteristic microstructural pattern of the main brain tissues. In fact, we obtained a high value for $\nu_{i a}$ for the white matter (WM) compared to the gray matter (GM) or the cerebrospinal fluid (CSF) with the highest values in the single fibers areas like the corticospinal tract or the corpus callosum. As expected, both $\lambda_{\|}$and $\lambda_{\perp}$ reach the limit value of $3 \times 10^{-3} \mathrm{~mm}^{2} / \mathrm{s}$ in the CSF. While $\lambda_{\|}$conserve high values in basically all the WM and, in particular, in the single fiber areas. $\lambda_{\perp}$ values are generally very low in WM, in particular in the brain region where $\lambda_{\|}$ is high. In general, the results of our neural network trained on synthetic data are coherent with the results obtained by fitting multi-compartmental models present in the literature [2, 3, 4].

\section{DISCUSSION AND CONCLUSION}

In this work, we investigate for the first time how the dMRI signal representation chosen as an input for FC-NN impacts

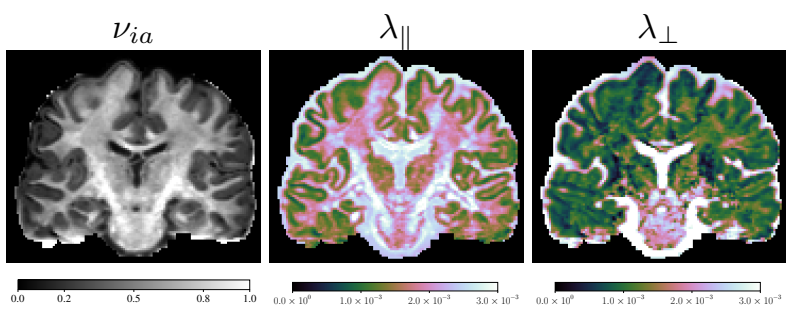

Fig. 2. In-vivo microstructural parameters estimation obtained on one HCP subject for RIF-NET with $n_{h}=4$ and $n_{p}=128$, the best performing network on the synthetic dataset.

the estimation of the most common brain microstructural features obtained from multi-compartmental models. Previous works on the use of FC-NN for estimating microstructural indices consider only the dMRI signal as an input for the network [6]. We showed that the use of a compact and meaningful representation like the novel RIF which we recently proposed in Zucchelli et. al. (2020) [4] is able to improve the performance of the neural networks in particular for small networks. This result can be explained considering that dMRI microstructural indices such as $\nu_{i a}, \lambda_{\|}$, and $\lambda_{\perp}$ are naturally rotation invariants. Giving the network an input that is rotation invariant increases the efficiency of the network itself that can use all its weights to estimate the desired output. This observation is sustained by the fact that the performance of both COEF-NET and SIG-NET tends to go nearer the one of SIGNET if the number of weights of the network increases. It is interesting to note that in the case of SIG-NET for example, simple rotation invariant features such as the spherical mean [2] can be easily obtained from the raw diffusion signal by the neural network itself. The increase of performance by SIG-NET and COEF-NET can be explained by the fact that the ability of the networks to extract RIF increases with the complexity of the networks themselves.

In this work, we only considered FC-NN that takes a single voxel signal representation as input and not the imageoriented convolutional neural networks $(\mathrm{CNN})$ that are commonly used in biomedical imaging [5]. The main advantage of our approach compared to the latter is the possibility to guide the network training using synthetic data for which it is possible to have ground truth values. In order to obtain the same results with image-oriented CNN, it is necessary to first compute the brain microstructural features on hundreds of subjects by fitting the chosen multi-compartment model and secondly to train the networks using the result of the model fitting, similarly to what has been done in [6]. This methodology is not only slower but also less accurate because the error in the model fitting are propagated to the CNN. This limits the maximum performance of the $\mathrm{CNN}$ to the one of the multicompartment model used for the initial fitting. On the contrary, using a synthetic dataset with ground truth values we 
are able to theoretically achieve the optimal performance as long as our training set is representative enough. The parameters distribution of the synthetic dataset should mimic the distribution of the microstructural parameters of a real brain. This is a challenge in itself and can be considered one of the main limitations of the FC-NN approach. Although we process the brain voxel-by-voxel and no neighborhood information is used by our FC-NN the resulting brain images (Figure 2 appear smooth and natural. There are no visible fitting artifact or discrepancy in the value of the microstructural indices for neighboring voxels.

In conclusion, the combination of our newly proposed RIF and FC-NN are able to provide an accurate estimation of brain microstructural indices. Our future works will focus on identifying the weight and importance that each single invariant carries on the microstructure estimation, the comparison of our method with other NN approaches such as the spherical convolution neural networks [10], and the application of our networks to clinical data.

\section{ACKNOWLEDGMENTS}

This work has been supported by the French government, through the 3IA Côte D'Azur Investments in the Future project managed by the National Research Agency (ANR) with the reference number ANR-19-P3IA-0002.

This work has received funding from the European Research Council (ERC) under the European Union's Horizon 2020 research and innovation program (ERC Advanced Grant agreement No 694665 : CoBCoM - Computational Brain Connectivity Mapping).

Data were provided by the Human Connectome Project, WU-Minn Consortium (Principal Investigators: David Van Essen and Kamil Ugurbil; 1U54MH091657) funded by the 16 NIH Institutes and Centers that support the NIH Blueprint for Neuroscience Research; and by the McDonnell Center for Systems Neuroscience at Washington University.

\section{COMPLIANCE WITH ETHICAL STANDARDS}

This research study was conducted retrospectively using human subject data made available in open access by (https: //www.humanconnectome.org/). Ethical approval was not required as confirmed by the license attached with the open access data.

\section{REFERENCES}

[1] H. Zhang, Schneider T., C.A.N. Wheeler-Kingshott, and D.C. Alexander, "NODDI: Practical in vivo neurite orientation dispersion and density imaging of the human brain," Neuroimage, vol. 61, no. 4, pp. 1000-1016, 2012.
[2] Enrico Kaden, Nathaniel D. Kelm, Robert P. Carson, Mark D. Does, and Daniel C. Alexander, "Multicompartment microscopic diffusion imaging," $\mathrm{Neu}$ roImage, vol. 139, pp. 346 - 359, 2016.

[3] Dmitry S. Novikov, Jelle Veraart, Ileana O. Jelescu, and Els Fieremans, "Rotationally-invariant mapping of scalar and orientational metrics of neuronal microstructure with diffusion mri," NeuroImage, vol. 174, pp. 518 $-538,2018$.

[4] Mauro Zucchelli, Samuel Deslauriers-Gauthier, and Rachid Deriche, "A computational framework for generating rotation invariant features and its application in diffusion mri," Medical Image Analysis, vol. 60, pp. 101597, 2020.

[5] Tyler Clark, Alexander Wong, Masoom A. Haider, and Farzad Khalvati, "Fully deep convolutional neural networks for segmentation of the prostate gland in diffusion-weighted mr images," in Image Analysis and Recognition, Fakhri Karray, Aurélio Campilho, and Farida Cheriet, Eds., Cham, 2017, pp. 97-104, Springer International Publishing.

[6] V. Golkov, A. Dosovitskiy, J. I. Sperl, M. I. Menzel, M. Czisch, P. Smann, T. Brox, and D. Cremers, "q-space deep learning: Twelve-fold shorter and model-free diffusion mri scans," IEEE Transactions on Medical Imaging, vol. 35, no. 5, pp. 1344-1351, 2016.

[7] Herbert H.H. Homeier and E.Otto Steinborn, "Some properties of the coupling coefficients of real spherical harmonics and their relation to gaunt coefficients," Journal of Molecular Structure: THEOCHEM, vol. 368, pp. 31 - 37, 1996, Proceedings of the Second Electronic Computational Chemistry Conference.

[8] Emmanuel Caruyer and Ragini Verma, “On facilitating the use of hardi in population studies by creating rotation-invariant markers," Medical image analysis, vol. 20, no. 1, pp. 87-96, 2015.

[9] Stamatios N Sotiropoulos, Saad Jbabdi, Junqian Xu, Jesper L Andersson, Steen Moeller, Edward J Auerbach, Matthew F Glasser, Moises Hernandez, Guillermo Sapiro, Mark Jenkinson, et al., "Advances in diffusion MRI acquisition and processing in the human connectome project," Neuroimage, vol. 80, pp. 125-143, 2013.

[10] Sara Sedlar, Théodore Papadopoulo, Rachid Deriche, and Samuel Deslauriers-Gauthier, "Diffusion MRI fiber orientation distribution function estimation using voxelwise spherical U-net," in Computational Diffusion MRI, MICCAI Workshop, Lima, Peru, Oct. 2020. 